\title{
Agatolimod Sodium
}

National Cancer Institute

\section{Source}

National Cancer Institute. Agatolimod Sodium. NCI Thesaurus. Code C28580.

The tricosasodium salt of a synthetic 24-mer oligonucleotide containing 3 CpG motifs with potential antineoplastic and immunostimulatory activity. Agatolimod selectively targets Toll-like receptor 9 (TLR9), thereby activating dendritic and B cells and stimulating cytotoxic T cell and antibody responses ag ainst tumor cells bearing tumor antigens. 